\title{
Seminome: Geringere Belastung durch risikoadaptierte Therapie
}

\author{
Ziel der vorliegenden Studie war es, den Nutzen eines risikoadaptierten \\ Therapieprotokolls bei Patienten mit Seminomen im klinischen Stadium I \\ zu untersuchen. Dabei wurde eine adjuvante Carboplatin-Therapie mit der \\ alleinigen Beobachtung verglichen.
}

$B^{\mathrm{e}}$ ei Patienten mit Stadium-I-Seminomen gilt aufgrund von Ergebnissen der Swedish and Norwegian Testicular Cancer Group (SWENOTECA) Carboplatin adjuvant als Standard. Mit der prospektiven, populationsbasierten Studie SWENOTECA VII wurde 2007 ein risikoadaptiertes Therapieprotokoll eingeführt, das bei Hochrisikopatienten das Risiko von Nebenwirkungen der Salvagetherapie reduzieren und die $\mathrm{Be}$ handlungsbelastung minimieren sollte.

897 Patienten mit einem Stadium-ISeminom wurde bei Vorliegen der beiden Risikofaktoren für ein Rezidiv Stromainvasion im Rete testis und Tumordurchmesser $>4 \mathrm{~cm}$ - eine adjuvante Carboplatin-Therapie empfohlen, bei keinem oder nur einem Risikofaktor lediglich die Beobachtung. Unabhängig von der Empfehlung konnten die Patienten aber frei für Carboplatin oder Beobachtung entscheiden.

Während der medianen Beobachtungsdauer von 5,6 Jahren kam es bei 69 Patienten zu Rezidiven. Eine Stromainvasion im Rete testis (Hazard Ratio [HR] 1,9; $\mathrm{p}=0,011$ ) und ein Tumordurchmesser $>4 \mathrm{~cm}($ HR 2,7; $<<0,001)$ wurden als Risikofaktoren für ein Rezidiv bestätigt.

Bei Patienten ohne diese Faktoren betrug die Rezidivrate $4,0 \%$ unter alleiniger Beobachtung und 2,2\% unter adjuvantem Carboplatin. Bei Patienten mit 1 bzw. 2 Risikofaktoren lagen die Rezidivraten bei 15,5 versus $9,3 \%$. Die Carbo-
platin-Dosis hatte keinen signifikanten Einfluss auf das Rezidivrisiko.

Fazit: In dieser großen populationsbasierten Studie zeigte sich, dass die Therapie bei Patienten mit Seminomen im klinischen Stadium I risikoadaptiert erfolgen kann und sich die Zahl der Patienten, die eine Salvagetherapie benötigen, reduziert. Eine Stromainvasion im Rete testis und ein Tumordurchmesser $>4 \mathrm{~cm}$ sind bei Patienten mit Seminomen im klinischen Stadium I Risikofaktoren für ein Rezidiv. Patienten ohne diese Risikofaktoren haben ein niedriges Rezidivrisiko, sodass die adjuvante Therapie bei ihnen nicht gerechtfertigt ist. Judith Neumaier

Tandstad T et al. Treatment of stage I seminoma with one course of adjuvant carboplatin or surveillance, risk-adapted recommendations implementing patient autonomy: a report from the Swedish and Norwegian Testicular Cancer Group (SWENOTECA). Ann Oncol. 2016;27(7):1299-304.

\section{Nierenkrebs: Bei T1-Tumoren nur Teilresektion}

Die Publikation der EORTC-30904-Studie im Jahr 2010 befeuerte die Diskussion, ob bei Nierenzellkarzinomen (RCC) im Stadium T1 eine partielle Nierenresektion ausreicht oder ob die Niere doch besser im Ganzen entfernt werden sollte. Kanadische Urologen stellten beide Methoden erneut gegenüber.

$\mathrm{D}$ ie kanadischen Urologen werteten die Daten von 2.385 Patienten aus, die sich zwischen 2000 und 2015 aufgrund eines RCC im Frühstadium (T1) entweder einer radikalen (RN) oder einer partiellen Nephrektomie (PN) unterziehen mussten und die im Canadian Kidney Cancer System registriert waren. Dabei lag das Augenmerk besonders auf der Zeit bis zur Progression und der Nierenfunktion. Das mediane Follow-up betrug in der Gruppe mit RN 2,87 Jahre, in der mit PN 1,99 Jahre. Hinsichtlich Patientencharakteristika, Tumorhistologie und Tumorgröße sowie Nierenfunktion unterschieden sich die beiden Gruppen nicht signifikant.
Zum Auswertungszeitpunkt waren 32 Patienten in der PN-Gruppe und 58 in der RN-Gruppe verstorben. Von den Überlebenden hatten 86 (5\%) partiell und $76(10 \%)$ radikal nephrektomierte Patienten ein Rezidiv. Dabei fand sich bei der Zeit bis zur Progression kein signifikanter Unterschied zwischen beiden Gruppen ( $p=0,42)$, auch nicht bei Berücksichtigung potenzieller Einflussfaktoren. Progressionsfrei waren nach einem Jahr 98,0\% der PN-Patienten und 94,7 \% der RN-Patienten. Nach 3 Jahren lag der Anteil bei 97,8 bzw. 91,2\%. Die Patienten, die weniger radikal operiert worden waren, schnitten allerdings hinsichtlich ihrer Nierenfunktion etwas besser $a b$.
Fazit: Ob bei einem RCC im Stadium T1 die Niere ganz entfernt oder nur teilreseziert wird, mache für das progressionsfreie Überleben keinen Unterschied, schlussfolgern die kanadischen Urologen. Damit stünden die Ergebnisse dieser Auswertung - wie auch die zahlreicher anderer Studien - im Widerspruch zu denen der EORTC-30904-Studie, die einen kleinen Vorteil für RN-Patienten hinsichtlich des Gesamtüberlebens gefunden hatte. Als entscheidend für den Krankheitsverlauf hat sich in der kanadischen Untersuchung weniger das Operationsverfahren als vielmehr andere Faktoren herauskristallisiert, darunter vor allem die Tumorgröße (T1a vs. T1b), das Jahr der Diagnose, der histologische Subtyp sowie der Tumorgrad.

Dagmar Kraus

Forbes CM et al. Disease progression and kidney function after partial vs. radical nephrectomy for T1 renal cancer. Urol Oncol. 2016 Jul 13. [Epub ahead of print]. 